\title{
IMPACTOS DA PISCICULTURA EM TANQUES REDE SOBRE O AMBIENTE E ASSEMBLEIAS DE PEIXES RESIDENTES NO RESERVATÓRIO DE BALBINA, NA AMAZÔNIA CENTRAL
}

\section{IMPACTS OF THE FISH-FARMS IN CAGES ON THE WATER QUALITY AND ICHTHYOFAUNA OF THE BALBINA HYDROELECTRIC RESERVOIR, CENTRAL AMAZON}

\author{
Adry Thiago de Lima Trindade ${ }^{1}$, Hélio Beltrão ${ }^{2 *}$, Cidiane Melo Oliveira ${ }^{4}$, Bruce Rider Forsberg ${ }^{3}$ \& \\ Raniere Garcez Costa Sousa ${ }^{4 *}$ \\ ${ }^{1}$ Curso Superior de Tecnologia em Produção Pesqueira, Universidade do Estado do Amazonas - UEA \\ ${ }^{2}$ Pós-Graduação em Ciências Pesqueiras nos Trópicos, Universidade Federal do Amazonas-Ufam \\ ${ }^{3}$ Coordenação de Pesquisas em Clima e Ambiente, Instituto Nacional de Pesquisas da Amazônia - Inpa \\ ${ }^{4}$ Departamento de Engenharia de Pesca, Programa de Pós-Graduação em Ciências Ambientais, Universidade Federal \\ de Rondônia - Unir \\ *Autor correspondente: ranieregarcez@unir.br
}

RESUMO O presente estudo avaliou os impactos da piscicultura em tanques rede sobre a qualidade da água e sobre a ictiofauna do Reservatório da Usina Hidrelétrica (UHE) de Balbina, no município de Presidente Figueiredo, estado do Amazonas (Brasil). Foram avaliados os parâmetros físico-químicos da água (temperatura, oxigênio dissolvido, $\mathrm{pH}$, condutividade elétrica, carbono orgânico dissolvido, fósforo total e nitrogênio total), além da profundidade, transparência da água e estrutura das assembleias de peixes, durante os meses de fevereiro a maio de 2013, em um sitio controle e outro onde estavam instalados 8 tanques redes de $10 \mathrm{~m} 3$ com tambaquis (Colossoma macropomum), com densidade de estocagem de 50 peixes/m3. Os resultados revelaram que não houve influência da piscicultura em tanques rede sobre a qualidade de água. Contudo, ocorreu uma maior riqueza de espécies e composição das assembleias de peixes no sitio dos tanques rede, quando comparado ao sitio controle. As análises preliminares revelam que, a prática de piscicultura em tanques rede, em baixa escala, no lago de Balbina, não apresentou aumentos significativos nas variáveis indicadoras de eutrofização, contudo, essa prática pode interferir na estrutura populacional das espécies de peixes residentes se os cultivos ocorrerem por períodos prolongados.

Palavras-chave: Colossoma macropomum, produção de peixes, qualidade da água, espécies nativas

\begin{abstract}
The summary of this body text should be written The present study evaluated the impacts of fishfarms in cages on water quality and on the ichthyofauna of the hydroelectric reservoir of Balbina, in the municipality of Presidente Figueiredo, Amazonas State. The physical-chemical parameters of the water characteristics (temperature, dissolved oxygen, $\mathrm{pH}$, electrical conductivity, dissolved organic carbon, total phosphorus and total nitrogen), besides of the depth and transparency of the water, and assemblages fish structure were evaluated, during the months of february/may of 2013, in a control site and in another where 8 cages of 10 $\mathrm{m} 3$ installed with tambaquis (Colossoma macropomum), with $50 \mathrm{fish} / \mathrm{m} 3$ density. The results show that no had influence of fish-farming on water quality. However, had on major richness and ichthyofauna composition in cages site when compared to the control site. Our preliminary analyses reveal that, the practice of fish-farming in small scale on Balbina Lake, not show significant increases in eutrophication indicator variables, however, this practice certainly can interfere in the population structure of resident fish species, if the fish farming remain for longer periods of time.
\end{abstract}

Keywords: Colossoma macropomum, fish production, water quality, native species. 


\section{Introdução}

A utilização dos cursos de rios para a construção de barragens, proporciona a formação de grandes lagos artificiais, que por sua vez, produzem diferentes impactos nos ambientes aquáticos e terrestres, principalmente com a alteração na mudança dos padrões físicos-químicos da água (Fearnside, 2012), dentre outros impactos relacionados, estão a remoção de comunidades humanas das áreas a serem inundadas, e perda da vegetação adjacente que fica submersa ocasionando a morte de grandes áreas de florestas, assim como a perda da biodiversidade em toda a área alagada pelo represamento (Tundisi \& Matsumura-Tundisi, 2003; Winemiller et al., 2016; Lees et al., 2016). Essa fase é uma das mais críticas, pois, a matéria orgânica em decomposição, provoca a eutrofização das áreas alagadas, que contribui com a produção de algas e macrófitas, ocasionando na maioria das vezes ao ambiente aquático o estado de anóxia, que se agrava com os gases de efeito estufa que são produzidos e emitidos em larga escala por períodos prolongados (Fearnside, 2012; 2015).

Contrário ao cenário de degradação ambiental, as áreas inundadas pelas águas represadas, formam grandes extensões que podem ser utilizadas na atividade de piscicultura, sobretudo com o emprego de tanques rede. Esta atividade esta em expansão em todo o mundo (FAO, 2017). No Brasil, a produção de pescado anualmente é de aproximadamente 1 milhão de toneladas, sendo a atividade aquícola responsável por mais de $30 \%$ deste total (Mallasen et al., 2012). A expansão da piscicultura se deve principalmente pela diminuição dos estoques pesqueiros naturais, em todo o mundo, o que tem incentivado o uso dessa prática de produção como alternativa viável para suprir a procura de proteína animal, ao mesmo tempo, contribuem com a preservação dos estoques de peixes em seus habitats naturais (Carvalho \& Ramos, 2010; Brabo et al., 2014).

O cultivo de peixes em tanques rede é uma modalidade de criação intensiva, que utiliza elevada densidade de estocagem e, por isso, exige constante renovação de água para a dispersão dos resíduos metabólicos deixados pelos peixes no ambiente (Ono \& Kubitza, 2003), que por sua vez, aumentam as concentrações de nitrogênio e fósforo na água (Guarino et al., 2005; Guo et al., 2009) promovendo a eutrofização ambiental, o que pode inviabilizar a prática da piscicultura nesses locais. No entanto, os impactos ambientais relacionados com a atividade piscícola intensiva sobre o ecossistema aquático de áreas represadas, e da ictiofauna residente, ainda não foram totalmente elucidados (Agostinho et al., 2007; Carvalho \& Ramos, 2010), principalmente em regiões ricas em biodiversidade aquática como a Amazônia.

Alguns estudos realizados nas bacias do médio Tietê e alto Paranapanema (no rio Paraná, Sudeste do Brasil) demonstraram que a piscicultura em tanques rede tem afetado em diferentes formas a ictiofauna residente, onde apresentaram maior diversidade de espécies no entorno das estruturas de criação (Paes, 2006; Carvalho et al., 2008), alterando principalmente as modificações no hábito alimentar e nas variáveis de crescimento (peso e comprimento) dos indivíduos das assembleias de peixes existentes nessas áreas (Ramos et al., 2008b), fatores estes atribuídos principalmente a dispersão de ração, que não são aproveitadas pelos peixes cultivados, e chegam a aproximadamente $30 \%$ do total empregado nos sistemas de criação, podendo contribuir, também, no processo de eutrofização ambiental (Bueno et al., 2011).

$\mathrm{Na}$ bacia Amazônica, a produção de peixes em tanques rede vem crescendo consideravelmente nos últimos anos, particularmente nos estados do Amazonas, Rondônia e Pará (Ono, 2005). Estudos mostram que este sistema de cultivo pode ser mais viável que os sistemas tradicionais (viveiros e barragens) considerando os aspectos econômico, técnico e social (Ono \& Kubitza, 2003), apresentando vantagens por não promover desmatamento de novas áreas, aproveitar o transporte fluvial, não depender de energia elétrica e agregar a produção de vários empreendimentos, além de apresentar a possibilidade de receitas econômicas para as populações locais (Brabo et al., 2014).

No entanto, mesmo com abundância de recursos hídricos propícios para a criação de peixes na região Amazônica (Ono, 2005), o principal problema para a implantação desse sistema de produção na região é a ausência de estudos sobre os impactos causados por estes empreendimentos, sobre o ambiente aquático e sua ictiofauna residente. Nesse sentido, visando compreender essa problemática nesse sistema de produção, o presente estudo teve como objetivo principal, analisar se o emprego dos tanques rede nas áreas de represa de uma hidrelétrica na Amazônia, influenciam a qualidade da água e as assembleias de peixes local. 


\section{Material e Métodos}

\section{ÁREA DE ESTUDO}

O estudo foi desenvolvido no reservatório da Hidrelétrica de Balbina, estado do Amazonas que possui uma área de aproximadamente $2.360 \mathrm{~km}^{2}$, situado no rio Uatumã, no estado do Amazonas, a cerca de $180 \mathrm{~km}$ da capital do estado, Manaus. Para o estudo foram selecionadas duas áreas: sitio 1, na margem esquerda do reservatório $\left(01^{\circ} 54^{\prime} 54,6^{\prime \prime} \mathrm{S}\right.$ e $\left.59^{\circ} 27^{\prime} 68,8^{\prime \prime} \mathrm{W}\right)$, onde foram instalados os tanques rede, e sitio 2 (área controle), na margem direita do lago $\left(01^{\circ} 54^{\prime} 37,8^{\prime \prime} \mathrm{S}\right.$ e $\left.059^{\circ} 29^{\prime} 32,2^{\prime \prime} \mathrm{W}\right)$, sem interferência da piscicultura, ambos separados cerca de $3,6 \mathrm{~km}$, e morfologicamente semelhantes na composição da paisagem (Figura 1).

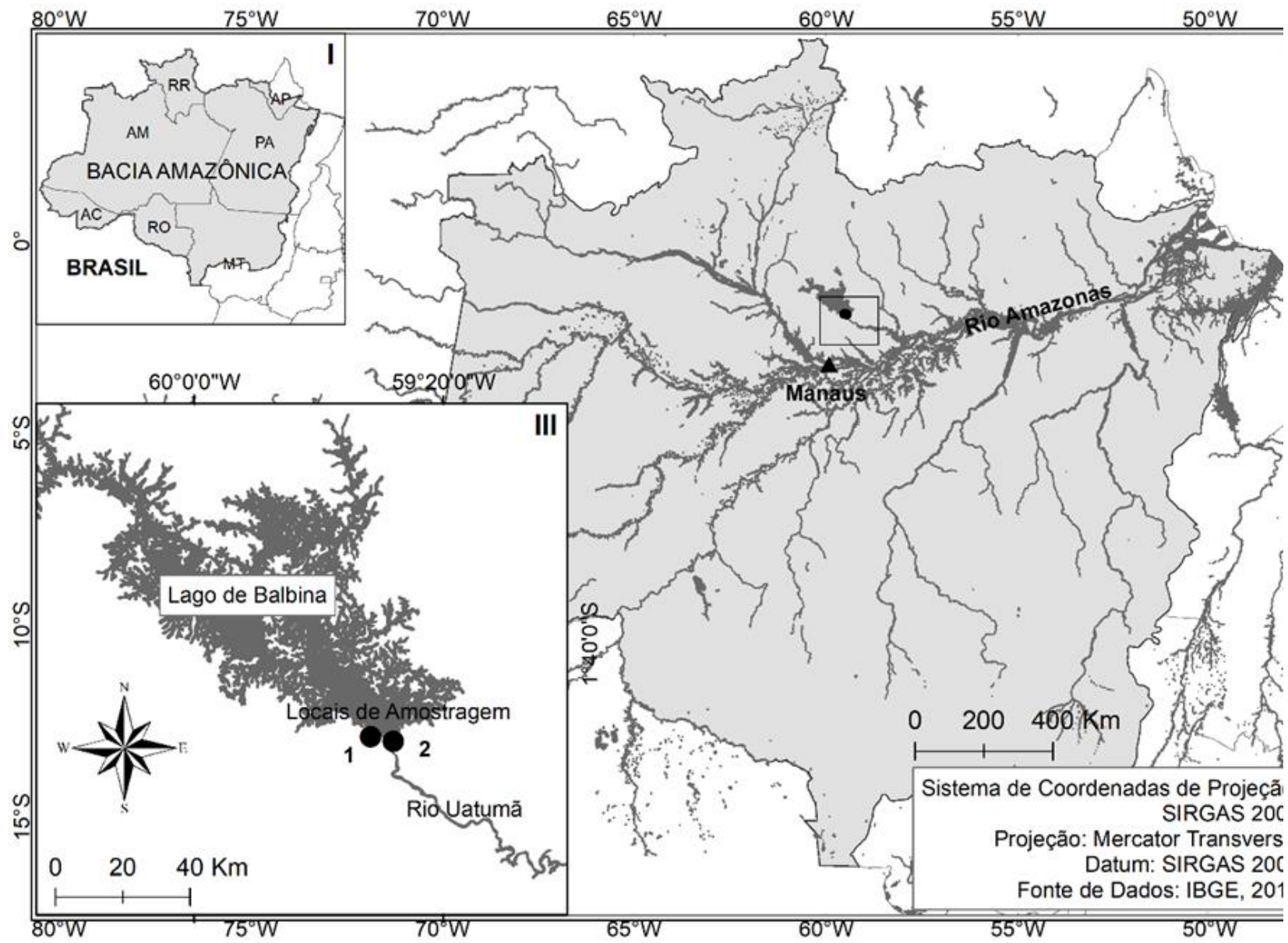

Figura 1. Mapa da porção Norte do Brasil, com destaque para o lago da Hidrelétrica de Balbina, estado do Amazonas e os respectivos sitios de amostragens, onde 1 representa o local da instalação dos tanques rede, e 2 o local do sitio controle (Fonte: IBGE, 2017)

\section{MONITORAMENTO DA CRIAÇÃO DE PEIXES NOS TANQUES-REDE}

Para investigar os impactos da piscicultura dos tanques-rede sobre a qualidade da água da área do reservatório, onde foi implantado os tanques, levantamentos de parâmetros físico-químicas da água foram realizados durante o período do estudo. Foram utilizados 8 tanques rede de $10 \mathrm{~m}^{3}(2,5 \times 2,5 \times 1,6 \mathrm{~m})$ organizados em duas fileiras, demandando uma área de aproximadamente 0,25 ha de lâmina d'água. A densidade de estocagem foi de 50 peixes $/ \mathrm{m}^{3}$, totalizando 4.000 indivíduos nos 8 tanques rede. A espécie utilizada no cultivo foi o Colossoma macropomum (tambaqui), com peso inicial médio de 176g (cada). Os peixes foram alimentados com ração peletizada, com $36 \%$ de proteína bruta, inicialmente fornecidas no equivalente de $5 \%$ da biomassa total (BT) dos peixes, e posteriormente reduzida a $3 \%$. Durante a pesquisa (fevereiro, abril e maio de 2013) foram fornecidos um total de $4.288 \mathrm{~kg}$ de ração para a alimentação dos peixes confinados.

ANÁLISES DAS VARIÁVEIS FÍSICO-QUÍMICAS DA ÁGUA E PARÂMETROS AMBIENTAIS 
Foram realizadas medidas de parâmetros físico-químicos da água em três pontos equidistantes em cada sitio amostral, com amostras na superfície da água (20 cm da coluna d’água) e no fundo $(50 \mathrm{~cm}$ próximo ao fundo). No sitio 1 (tanques rede), as análises foram realizadas nas duas extremidades dos tanques e na parte central. Já no sitio 02 (controle) foram analisados três pontos equidistantes, com a mesma metodologia empregada no sitio 1. Foram analisados ainda a profundidade (em metros) e a transparência da água (com disco de Secchi).

As variáveis físico-químicas da água medidas foram, temperatura e oxigênio dissolvido (oxímetro portátil YSI, Inc), condutividade elétrica e o pH (instrumento Orion Inc., 3-Star). Amostras de água foram armazenadas, para posteriores análises em laboratório do Carbono Orgânico Dissolvido (COD), com o auxílio de um Analisador de Carbono TOC (Shimadzu, Inc). Além disso, análises dos níveis de Fósforo total e Nitrogênio total, foram determinadas seguindo o Manual Micronal, utilizando um espectrofotômetro UV/Vível (Shimadzu, Inc.).

Análises dos parâmetros ambientais foram realizadas para verificar possíveis estratificação térmica ao longo da coluna d'água nos sítios amostrados. Neste caso, foi empregado um conjunto de sensores limnológicos que foram montados em um único ponto central, em cada sitio amostral. Os sensores limnológicos (Onset, Inc. U22-001 e U26-001) coletaram informações sobre temperatura e oxigênio dissolvido da água a cada $1,0 \mathrm{~m}$, entre a profundidade de $0,5 \mathrm{~m}$ até 7,5 $\mathrm{m}$. Todos os sensores coletaram informações em intervalos de 5 minutos durante 24 horas, todos os dias do experimento, isso serviu para caracterizar o padrão diel de estratificação de temperatura e oxigênio dissolvido tanto em padrões verticais como horizontais.

\section{COLETA DA ICTIOFAUNA}

Em cada sitio, foram realizadas três coletas de peixes, ao longo do período amostral, onde foi utilizado uma bateria de rede de espera, composta por 8 redes ( $10 \mathrm{~m}$ de comprimento, $2 \mathrm{~m}$ de altura e malhas variando de 20 a 90 mm entre nós opostos). As pescarias foram realizadas nos horários de 04:00-08:00hs e das 16:0020:00hs. Os espécimes coletados foram conservados em solução de formol a 10\%, para posterior identificação em laboratório do INPA, sendo mensurados as medidas de comprimento padrão $(\mathrm{cm})$ e o peso total (gramas) de cada indivíduo.

\section{ANÁLISE ESTATÍSTICA DOS DADOS}

Para as assembleias de peixes foram analisados os descritores ecológicos usando medidas de abundância absoluta $(\mathrm{N})$, riqueza de espécies $(\mathrm{S})$, diversidade de Shannon-Wiener (H'), equitabilidade (J') e dominância (d) (Magurran, 1988; Krebs, 1989). A frequência de ocorrência de cada espécie foi calculada pela porcentagem em que a espécie ocorreu em cada sitio durante o período amostral. Uma lista de espécies foi organizada em forma de tabela, para apresentar os resultados e seus índices ecológicos.

Para verificar as diferenças dos parâmetros físico-químicos da água entre os sitios, períodos de coletas e profundidades, foram realizados Análises de Variância (Anova) e teste T (Zar, 2009). Foi utilizado uma Análise de componentes principais (PCA) de forma a ordenar os sitios de coletas, em função dos parâmetros que caracterizam a qualidade da água, com o intuito de identificar quais variáveis limnológicas poderiam estar influenciando a qualidade da água entre os sitios. Para facilitar a compreensão, o gráfico de ordenações foi separado em dois diagramas, um com a ordenação das variáveis ambientais e físico químicas da água, e outro com a dos sítios (tanques rede e controle).

Para avaliar a composição das assembleias de peixes dos sitios, foi empregado o método de escalonamento multidimensional não métrico (NMDS), com o uso de um índice de similaridade de Jaccard (presença-ausência de espécies) entre os dois sitios ao longo do período amostral (Krebs, 1989). Para verificar as relações entre as características físico-químicas da água (representadas pelos eixos 1 e 2 da PCA) com a composição de espécies de peixes (representada pelos eixos 1 e 2 do NMDS), foram feitas regressões lineares simples utilizando os escores dos eixos 1 e 2 de ambas as análises (PCA x NMDS).

\section{Resultados}




\section{QUALIDADE DA ÁGUA}

As variáreis físico-químicas da água medidos na superfície, não apresentaram diferenças significativas entre os sitios de amostragens, durante o período de estudo. Por outro lado, os mesmos parâmetros quando avaliados entre a superfície e o fundo do reservatório, exibiram diferenças significativas para os valores de temperatura e oxigênio dissolvido, onde estes apresentaram os maiores valores na superfície (Tabela 1).

A profundidade encontrada nos dois sitios, variou de $12 \mathrm{~m}$ em fevereiro a $15 \mathrm{~m}$ em maio, com média 13,5 $\mathrm{m}( \pm 1,5)$, não havendo diferenças significativas $(\mathrm{p}<0,491)$. Houve diferenças significativas para a transparência da água $(\mathrm{p}<0,05)$, que foi maior no sitio dos tanques rede, $3,17 \mathrm{~m}( \pm 0,15)$, quando comparado ao sitio controle, com média de $2,49 \mathrm{~m}( \pm 0,10)$.

Tabela 1. Valores médios dos parâmetros físico-químicos da água analisados entre os sítios 1 (tanques rede) e 2 (controle), seguidos dos seus respectivos valores estatísticos (p) obtidos pelo teste-t, durante o período amostral.

\begin{tabular}{lcccccc}
\hline Variáreis & $\begin{array}{c}\text { Sitio 1 } \\
\text { (superfície) }\end{array}$ & $\begin{array}{c}\text { Sitio 1 } \\
\text { (fundo) }\end{array}$ & $\mathrm{p}$ & $\begin{array}{c}\text { Sitio 2 } \\
\text { (superfície) }\end{array}$ & $\begin{array}{c}\text { Sitio 2 } \\
\text { (fundo) }\end{array}$ & $\mathrm{p}$ \\
\hline $\mathrm{pH}$ & 6,10 & 6,02 & 0,520 & 5,92 & 6,00 & 0,540 \\
$\mathrm{O}_{2}(\mathrm{mg} / \mathrm{l})$ & 5,55 & 4,50 & $0,005^{*}$ & 5,75 & 4,26 & $0,004^{*}$ \\
Cond. $(\mu \mathrm{S})$ & 8,73 & 9,71 & 0,066 & 9,06 & 9,33 & 0,130 \\
$\mathrm{~T}\left({ }^{\circ} \mathrm{C}\right)$ & 30,11 & 29,46 & $0,012^{*}$ & 30,5 & 29,4 & $0,010^{*}$ \\
$\mathrm{NT}(\mathrm{mg} / \mathrm{l})$ & 0,49 & 0,54 & 0,505 & 0,40 & 0,49 & 0,480 \\
$\mathrm{FT}(\mathrm{mg} / \mathrm{l})$ & 0,012 & 0,017 & 0,195 & 0,010 & 0,011 & 0,180 \\
$\mathrm{COD}(\mathrm{mg} / \mathrm{l})$ & 1,84 & 1,56 & 0,630 & 1,94 & 1,45 & 0,620 \\
\hline
\end{tabular}

$\mathrm{T}=$ temperatura, $\mathrm{NT}$ = nitrogênio total, FT = fósforo total, COD = carbono orgânico dissolvido.

*existiram diferenças significativas entre as médias amostrais.

A análise de componentes principais (PCA) empregada para caracterizar os sitios de amostragens através dos parâmetros de qualidade da água, revelou que os eixos 1 (PCA1) e 2 (PCA2) explicaram 78,3\% da variância do conjunto de dados analisados. As amostras realizadas nos dois sitios, durante os três períodos de análises encontram-se dispersas no espaço bidimensional do diagrama da PCA (Figura 2A). Os resultados demonstram que os sitios (tanques rede e controle) agruparam-se ao longo do período amostral, revelando que não existiram grandes diferenças dos parâmetros físico-químicos da água entre os dois sitios, embora, algumas variáveis tenham apresentado mudanças ao longo do período, essas mudanças foram semelhantemente em ambos os sitios (Figura 2A e 2B).

As amostras realizadas no período de fevereiro destacaram-se pela maior influência da condutividade elétrica e do fósforo total, sendo essas variáveis as que exibiram maiores correlações com este período para ambos os sitios, e variaram inversamente aos valores de temperatura da água e oxigênio dissolvido (Figura 2B). No período de abril, a temperatura, o $\mathrm{pH}$ e o nitrogênio total tiveram seus valores mais elevados, em ambos os sitios. Já as amostras realizadas no mês de maio foram influenciadas principalmente pelos valores de profundidade, oxigênio dissolvido e carbono orgânico dissolvido, no entanto, estes variaram inversamente aos valores de transparência da água (DS). Observou-se também, que os menores valores para a transparência da água ocorreram em maio, o que contribuiu para o isolamento desse período no espaço bidimensional (Figura 2B). Em geral, ao avaliar o agrupamento dos locais estudados para os sitios 1 e 2, observou-se que a segregação por período de coleta (fevereiro, abril e maio), indicam que os fatores hidroquímicos do reservatório variaram ao longo do período amostrado (Figura 2B). 

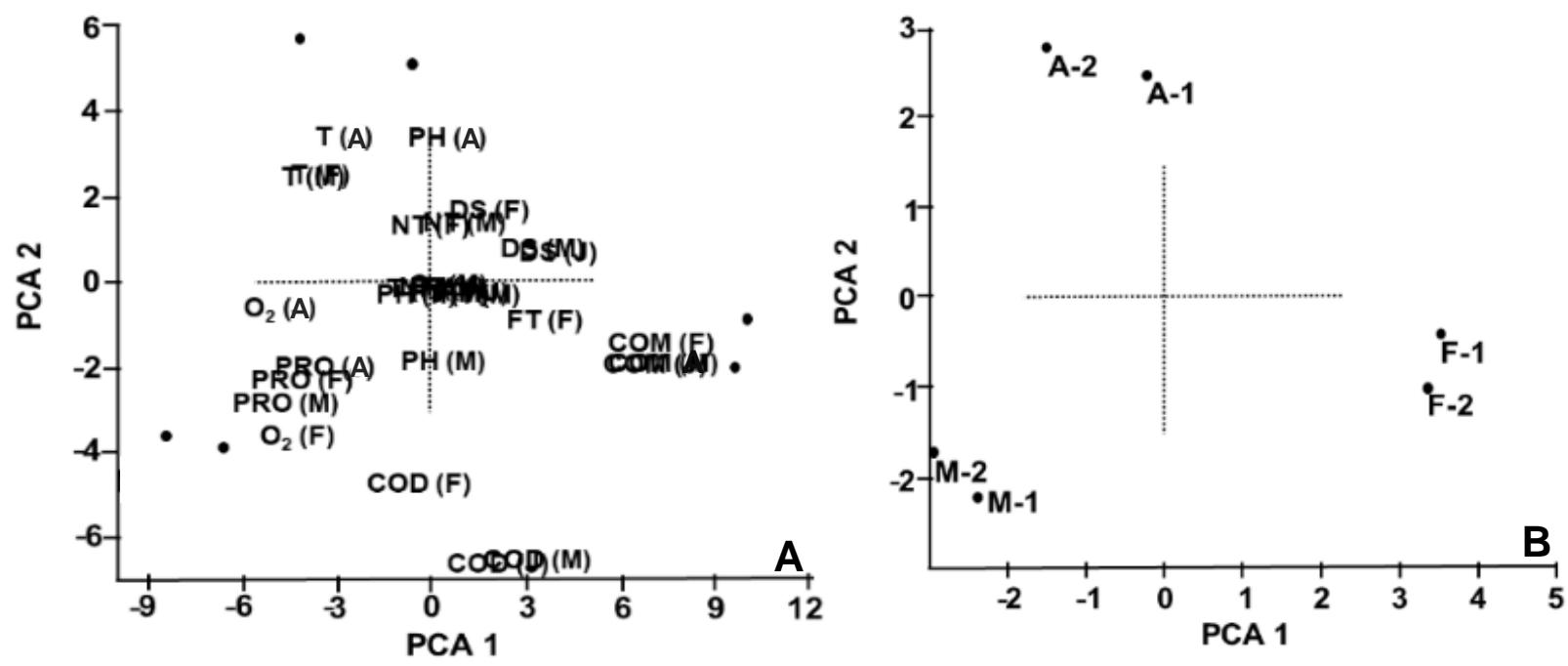

Figura 2. Projeções das variáveis físicas e físico-químicas da água (A) e as ordenações dos sitios de coletas, tanques rede (1) e controle (2) ao longo do período de estudo (B). Onde: $\mathrm{O} 2=$ Oxigênio dissolvido (mg/L), $\mathrm{T}=$ Temperatura da água $(\mathrm{oC}), \mathrm{PH}=$ Potencial Hidrogeniônico, $\mathrm{COM}=$ Condutividade elétrica $(\mu \mathrm{S}), \mathrm{COD}=$ carbono orgânico dissolvido $(\mathrm{mg} / \mathrm{L}), \mathrm{NT}=$ Nitrogênio total $(\mathrm{mg} / \mathrm{L})$, FT = Fósforo total $(\mathrm{mg} / \mathrm{L})$, PRO = Profundidade $(\mathrm{m})$ e DS = Transparência da água, $\mathrm{F}=$ Fevereiro, $\mathrm{A}=$ Abril e $\mathrm{M}=$ Maio.

\section{PADRÕES DE ESTRATIFICAÇÃO DE TEMPERATURA E OXIGÊNIO}

Os padrões de estratificação térmica e de oxigênio dissolvidos variaram durante um intervalo de 24 horas e entre os sitios amostrais (Figura 3), implicando na mistura da coluna de água, tanto no sentido vertical quanto horizontal ao longo do período diurno para ambos os sítios amostrais. Por outro lado, no sitio 1 a estratificação resultou num padrão fraco de temperatura (Figura 3A) e de oxigênio na coluna d'água (Figura 3C). Durante o período noturno, com o esfriamento da camada superficial, o padrão de estratificação termal foi reduzido gradativamente até a coluna se tornar isotermal, em geral entre as 3:00 às 7:00 horas, com média de $29,9 \mathrm{~T}^{\circ} \mathrm{C}$ (Figura 3A). Também no período noturno, em geral houve queda nas concentrações de oxigênio na camada superficial (entre 0,5 a 2,5 m) no sitio 1, principalmente entre as 21:00 horas até as 3:00 horas (Figura 3C), após esse período, houve mistura vertical, associada a estratificação isotermal e a geração de correntes de densidade vertical na coluna d'água.

Em geral, a queda dos níveis de oxigênio dissolvido durante o período de 19:00 as 3:00 horas, está associado ao consumo de oxigênio pelos organismos aquáticos, dentre eles os peixes cultivados. No entanto, o padrão de estratificação isotermal, aumentou os níveis de oxigênio após o horário das 3:00 hs (Figura 3C).

Um padrão de estratificação foi observado no sitio controle (Figura 3B e 3D). Em geral foram observadas estratificação de temperatura e de oxigênio dissolvido mais evidentes nesse sitio. Foi observado que a temperatura da água foi mais elevada no sitio 2 que no 1 ao longo do período diurno (Figura 3B), sendo o mesmo observado para os níveis de oxigênio na coluna d'água (entre 0,5 a 7,5m) (Figura 3D). Foi verificado que a temperatura variou ao longo do período diurno, mas os níveis de oxigênio na primeira camada da coluna d'água (entre 0,5 a 2,5m) permaneceram estáveis entre 7,5 a $7,7 \mathrm{mg} / \mathrm{L}$ ao longo de todo o período diurno (Figura 3D), havendo apenas oscilações nos níveis de oxigênio dissolvido após 3,5 $\mathrm{m}$ de profundidade (Figura 3D).

\section{CARACTERIZAÇÃO DA ICTIOFAUNA NOS AMBIENTES ESTUDADOS}

Foram capturadas 18 espécies de peixes, pertencentes a 3 ordens e 8 famílias, resultando em um total de 347 indivíduos coletados. Os Characiformes constituíram o grupo de maior riqueza, com 9 espécies (50,0\%), seguidos de Perciformes, com 5 espécies $(27,8 \%)$ e Siluriformes, com 4 espécies (22,2\%) (Tabela 2). Os Characiformes também constituíram o grupo de maior abundância $(n=268)$, seguidos por Perciformes $(n=$ 48). Já os Siluriformes apresentaram número reduzido de indivíduos (Tabela 2). 

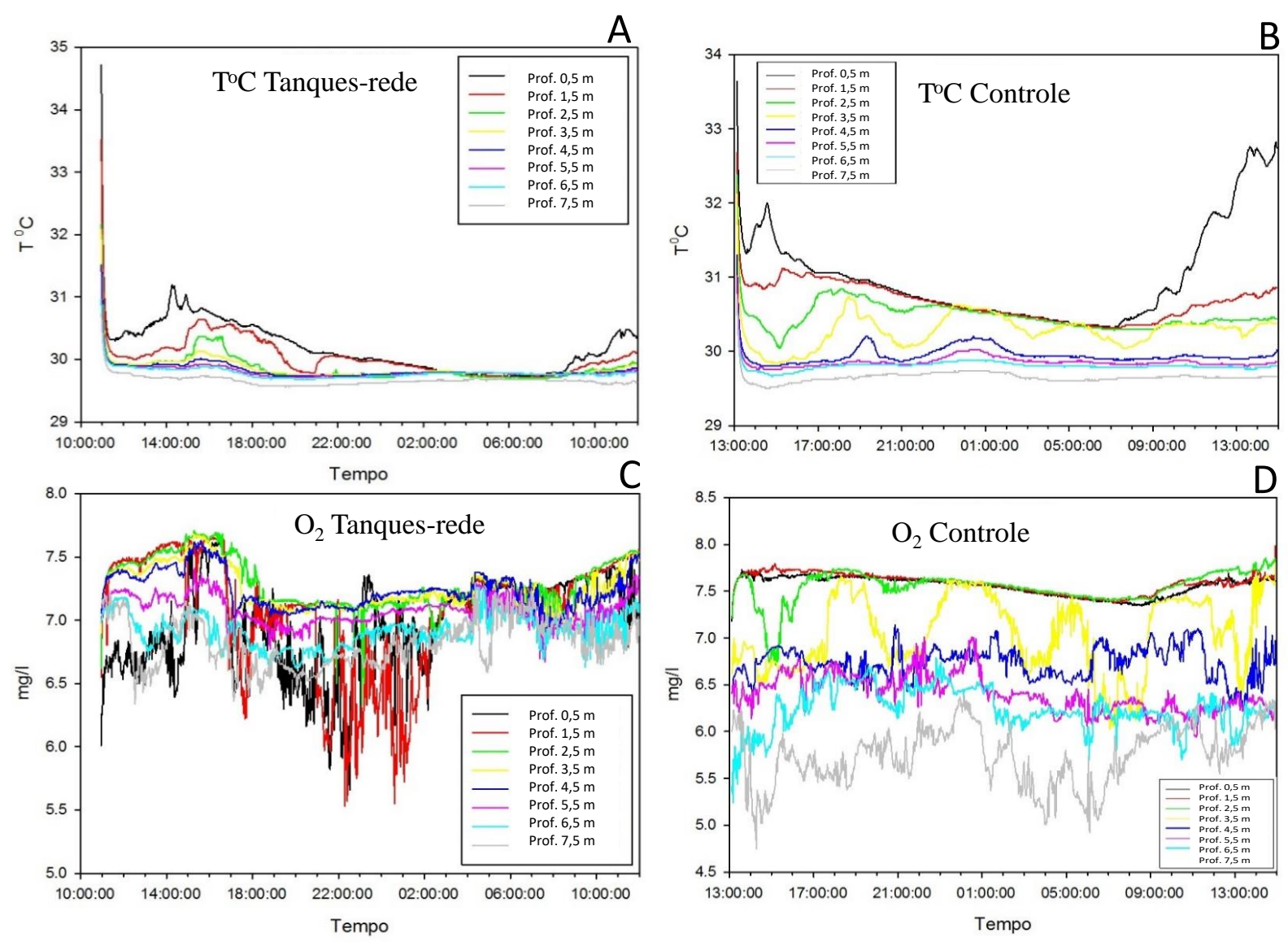

Figura 3. Padrão de variação da estratificação térmica e de oxigênio dissolvido nas profundidades de 0,5m a 7,5m, observadas nos sitios tanques rede (1) e controle (2).

A riqueza de espécies e o número de indivíduos variou entre os sitios de coletas. No sitio 1 foram capturados $262(75,5 \%)$ indivíduos pertencentes a 14 espécies, sendo as mais abundantes Bryconops alburnoides (33,2\%), Agoniates halecinus (22,5\%) e Metynnis argenteus (9,2\%). Já no sitio 2, foram capturados $85(24,5 \%)$ exemplares de 11 espécies, sendo os mais abundantes Agoniates halecinus $(29,4 \%)$, Serrasalmus rhombeus (24,7\%) e Plagioscion squamosissimus (20,0\%). Apenas 7 das 18 espécies foram comuns em ambos os sitios (Tabela 2).

A espécie Bryconops alburnoides foi a mais abundante no sitio 1, dentre outras de pequeno porte $(<10$ cm de comprimento padrão - CP), como a Bryconops giacopinii, Moenkhausia lepidura e Brycon pesu) que circulavam com frequência ao redor dos tanques rede, principalmente nos horários de alimentação dos tambaquis cultivados. Espécies maiores (20-50 cm de CP) como a Agoniates halecinus, Cichla monoculus, Plagioscion squamosissimus, Ageneiosus vittatus e Acestrorhynchus microlepis, também foram encontradas em maior número no sitio 1. Além destas espécies, Metynnis hypsauchen, Metynnis argenteus, Colossoma macropomum, Ageneiosus inermis e Geophagus altifrons, foram capturadas exclusivamente no sitio dos tanques rede (Tabela 2). No sitio 2 (controle), as espécies Leporinus granti, Pimelodus blochi, Cichla temensis e Crenicichla cf. lugubris foram exclusivas para o local (Tabela 2).

Em relação aos índices que estruturam a comunidade de peixes, houve variação tanto entre os sitios quanto entre os períodos de coletas. Os valores de diversidade de Shannon-Winner variaram entre $\mathrm{H}^{\prime}=0,53$ (sitio controle - abril) a H'=2,05 (sitio tanques rede - maio) (Tabela 3), onde exibiram uma maior dominância para as espécies Bryconops alburnoides e Agoniates halecinus no sitio 1 (Tabelas 2 e 3).

Tabela 2. Classificação taxonômica das espécies encontradas na área, Hidrelétrica de Balbina, estado do Amazonas. 


\begin{tabular}{|c|c|c|c|c|c|c|c|c|}
\hline Ordem/Família/Espécie & $\mathrm{F} 1$ & A1 & M1 & $\mathrm{F} 2$ & $\mathrm{~A} 2$ & M2 & Total & $\%$ \\
\hline \multicolumn{9}{|l|}{ CHARACIFORMES } \\
\hline \multicolumn{9}{|l|}{ ACESTRORHYNCHIDAE } \\
\hline Acestrorhynchus microlepis (Jardine 1841) & 1 & 0 & 1 & 0 & 0 & 0 & 2 & 0,58 \\
\hline \multicolumn{9}{|l|}{ ANOSTOMIDAE } \\
\hline Leporinus granti Eigenmann 1912 & 0 & 0 & 0 & 0 & 0 & 1 & 1 & 0,29 \\
\hline \multicolumn{9}{|l|}{ CHARACIDAE } \\
\hline Bryconops alburnoides Kner 1858 & 13 & 62 & 12 & 5 & 1 & 0 & 93 & 26,80 \\
\hline \multicolumn{9}{|l|}{ HEMIODONTIDAE } \\
\hline Hemiodus argenteus Pellegrin 1909 & 0 & 1 & 1 & 5 & 0 & 2 & 9 & 2,59 \\
\hline \multicolumn{9}{|l|}{ SERRASALMIDAE } \\
\hline Colossoma macropomum (Cuvier 1816) & 0 & 2 & 0 & 0 & 0 & 0 & 2 & 0,58 \\
\hline Metynnis argenteus Ahl 1923 & 0 & 8 & 16 & 0 & 0 & 0 & 24 & 6,92 \\
\hline Metynnis hypsauchen (Müller \& Troschel 1844) & 0 & 9 & 14 & 0 & 0 & 0 & 23 & 6,63 \\
\hline Serrasalmus rhombeus (Linnaeus 1766) & 0 & 4 & 5 & 14 & 0 & 7 & 30 & 8,65 \\
\hline \multicolumn{9}{|l|}{ TRIPORTHEIDAE } \\
\hline Agoniates halecinus Müller \& Troschel 1845 & 33 & 11 & 15 & 4 & 16 & 5 & 84 & 24,21 \\
\hline \multicolumn{9}{|l|}{ SILURIFORMES } \\
\hline \multicolumn{9}{|l|}{ AUCHENIPTERIDAE } \\
\hline Auchenipterichtys longimanus (Günther 1864) & 1 & 0 & 17 & 0 & 0 & 1 & 19 & 5,48 \\
\hline Ageneiosus vittatus Steindachner 1908 & 4 & 0 & 0 & 2 & 0 & 0 & 6 & 1,73 \\
\hline Ageneiosus inermis (Linnaeus 1766) & 2 & 0 & 1 & 0 & 0 & 0 & 3 & 0,86 \\
\hline \multicolumn{9}{|l|}{ PIMELODIDADE } \\
\hline Pimelodus blochi Valenciennes 1840 & 0 & 0 & 0 & 0 & 0 & 3 & 3 & 0,86 \\
\hline \multicolumn{9}{|l|}{ PERCIFORMES } \\
\hline \multicolumn{9}{|l|}{ CiCHLIDAE } \\
\hline Cichla monoculus Spix \& Agassiz 1831 & 1 & 0 & 4 & 0 & 0 & 0 & 5 & 1,44 \\
\hline Cichla temensis Humboldt 1821 & 0 & 0 & 0 & 0 & 0 & 1 & 1 & 0,29 \\
\hline Crenicichla cf. lugubris Heckel 1840 & 0 & 0 & 0 & 0 & 0 & 1 & 1 & 0,29 \\
\hline Geophagus altifrons Heckel 1840 & 10 & 1 & 1 & 0 & 0 & 0 & 12 & 3,46 \\
\hline \multicolumn{9}{|l|}{ SCIANIDAE } \\
\hline \multirow[t]{2}{*}{ Plagioscion squamosissimus (Heckel 1840) } & 9 & 2 & 1 & 2 & 2 & 13 & 29 & 8,36 \\
\hline & 74 & 100 & 88 & 32 & 19 & 34 & 347 & 100 \\
\hline
\end{tabular}

Quando: $\mathrm{F}=$ fevereiro, $\mathrm{A}=$ abril, $\mathrm{M}=$ maio, $1=$ sitio com os tanques rede, 2 = sitio controle.

Tabela 3. Descritores ecológicos da ictiofauna capturadas no lago de Balbina.

\begin{tabular}{lccccccc}
\hline Descritores & $\mathrm{F}(1)$ & $\mathrm{A}(1)$ & $\mathrm{M}(1)$ & $\mathrm{F}(2)$ & $\mathrm{A}(2)$ & $\mathrm{M}(2)$ & Total \\
\hline Riqueza $(\mathrm{S})$ & 9 & 9 & 12 & 6 & 3 & 9 & 18 \\
Abundância (N) & 74 & 100 & 88 & 32 & 19 & 34 & 347 \\
Diversidade (H') & 1,62 & 1,33 & 2,05 & 1,55 & 0,53 & 1,77 & 2,17 \\
Equitabilidade (J) & 0,73 & 0,61 & 0,82 & 0,86 & 0,48 & 0,81 & 0,75 \\
Dominância (d) & 0,44 & 0,62 & 0,19 & 0,43 & 0,84 & 0,38 & 0,27 \\
\hline
\end{tabular}

Quando: $\mathrm{F}=$ fevereiro, $\mathrm{A}=$ abril, $\mathrm{M}=$ maio, $1=$ sitio tanques rede, $2=$ sitio controle.

A composição de espécies foi bastante heterogênea entre os sitios de coleta. A similaridade média (calculada pelo índice de Jaccard) entre os locais de amostragens foi de 0,35. Implicando que 35\% das espécies 
amostradas compartilharam os sítios. A maior similaridade de espécies foi registrada no sitio 1 entre os meses de abril e maio (Figura 4A). Em geral, a fauna de peixes do sitio 1 foi relativamente distinta daquela encontrada no sitio 2, observados pelo extremo distanciamento destes pontos no cladograma de Cluster (Figura 4A).

$\mathrm{Na}$ análise de Escalonamento Multidimensional Não-Métrico (NMDS), baseados também em informações de presença/ausência de espécies nos distintos períodos e sitios de coletas, revelaram a heterogeneidade das assembleias de peixes entre os dois sitios. Os dois primeiros eixos retidos para interpretação, conseguiram capturar grande parte da variação dos dados originais de composição das assembleias de peixes, como indicado pelos valores de configuração (Stress de Kruskal $=0,300$ ). As distribuições dos sítios de coletas indicam a presença de um aparente gradiente entre as assembleias de peixes, onde o sitio 1, com maior riqueza e similaridade na composição da ictiofauna, separa-se do sitio 2 , menos similares, evidenciado principalmente ao longo do eixo 2 (Figura 4B). O gráfico revela que as amostragens realizadas no sitio 1, parte inferior, apresenta uma ictiofauna mais similar, indicada pelos pontos agrupados no espaço bidimensional (Figura 4B) seguido das amostragens realizadas no sitio 2, porção superior, menos similar e com os pontos mais distantes (Figura 4B).

As análises de regressões lineares demonstraram que não houveram relações significativas entre os parâmetros de características da qualidade da água entre os sitios (representadas pelos escores dos eixos 1 e 2 da PCA) com a composição das assembleias de peixes (representadas pelos escores dos eixos 1 e 2 da NMDS), PCA1 x NMDS1 $\left(r=-0,559 ; r^{2}=0,312 ; p=0,248\right)$ e PCA1 x NMDS2 $\left(r=0,0071 ; r^{2}=0,000 ; p=0,989\right)$, demostrando que outros fatores estão influenciando na composição de espécies entre os sitios amostrais.
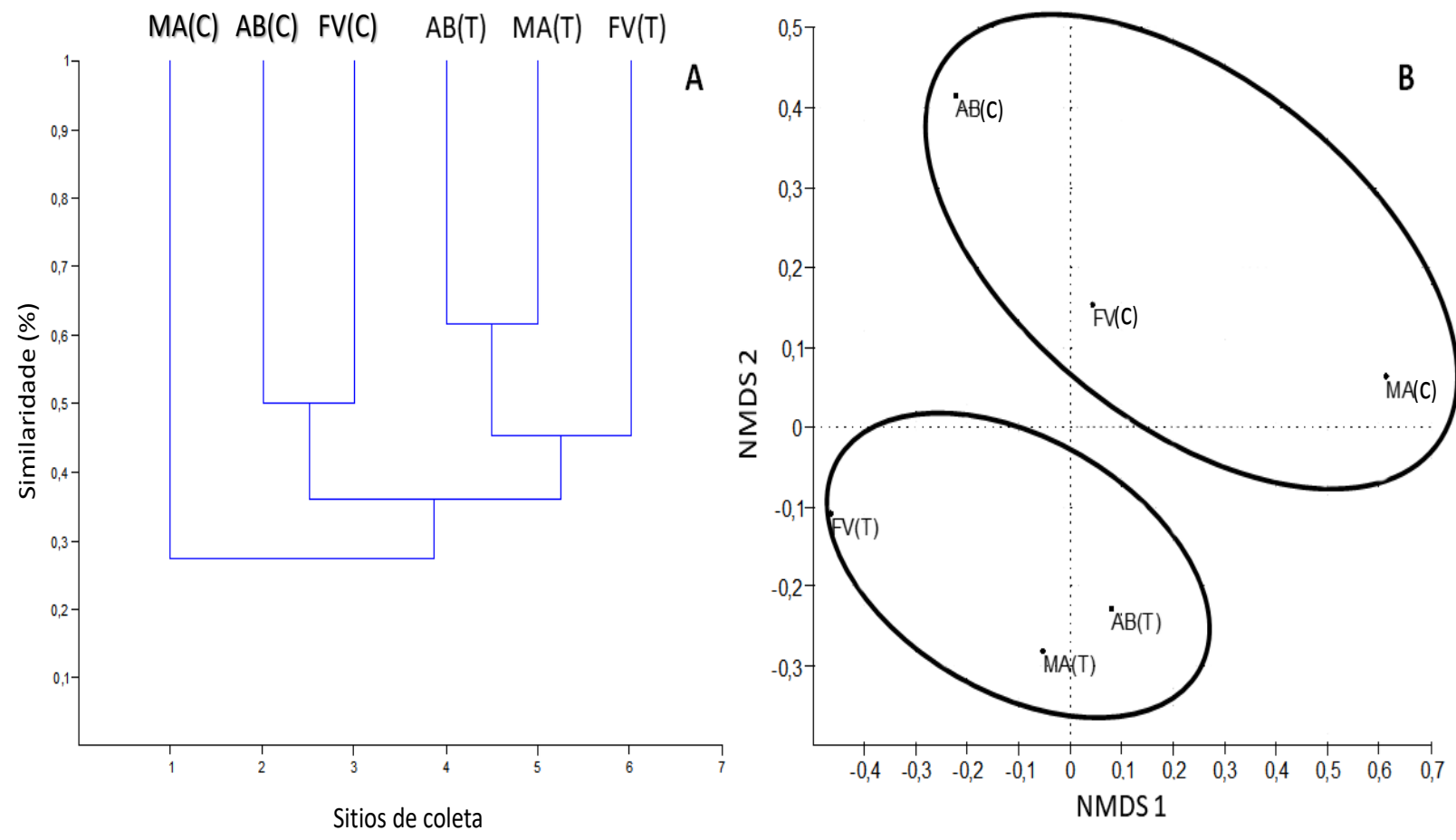

Figura 4. (A) Dendrograma de Similaridade de Jaccard referente a conjuntos de espécies de peixes registradas nos dois diferentes sitios. (B) Ordenamento das amostras de peixes por meio de Escalonamento Multidimensional Não-Métrico (NMDS), com base na composição de espécies. Os Círculos contínuos, referem-se aos sitios tanques rede e controle. Onde: $\mathrm{FV}(\mathrm{T})=$ fevereiro (tanques rede), $\mathrm{AB}(\mathrm{T})=$ abril (tanques rede), $\mathrm{MA}(\mathrm{T})=$ maio (tanques rede), $\mathrm{FV}(\mathrm{C})=\mathrm{fevereiro}$ (controle), $\mathrm{AB}(\mathrm{C})=$ abril (controle) e $\mathrm{MA}(\mathrm{C})=$ maio (controle).

\section{Discussão}


Durante o período de estudo, não foram observadas diferenças significativas entre os valores médios das variáveis limnológicas entre os sítios. Além disso, a maioria das variáveis limnológicas analisadas nos sitios estudados, permaneceram dentro dos níveis aceitáveis para a criação de peixes, segundo a resolução do Conama n⿳⺈-357, de 17 de março de 2005.

Quanto as variáveis indicadoras de eutrofização como o fósforo total e o nitrogênio total, os maiores valores de fósforos total foram encontrados no fundo do sitio com tanques rede $(0,017 \mathrm{mg} / \mathrm{L})$ quando comparado ao sitio controle $(0,011 \mathrm{mg} / \mathrm{L})$. Este incremento de fósforo, embora, não significativo, dentro do sistema, pode ser atribuído as sobras de rações e ao maior índice pluviométrico, carreando o elemento para o fundo do lago. Os valores de fósforos observados neste estudo estão abaixo dos valores máximos recomendado (Conama, 2005) que é de até $0,05 \mathrm{mg} / \mathrm{L}$, para água destinadas a aquicultura.

Para o nitrogênio total (NT, mg/L), os maiores valores também foram encontrados no fundo do sitio de tanques rede $(0,54 \mathrm{mg} / \mathrm{L})$. Estes valores também estão abaixo dos limites estabelecidos pela Resolução Conama No. $351 / 2005$ onde estabelece que estes não ultrapassem $1,27 \mathrm{mg} / \mathrm{L}$, em ambientes lênticos, quando o nitrogênio for fator limitante para eutrofização (Conama, 2005). O nitrogênio total é um metabólito proveniente da excreção nitrogenada dos peixes, bem como da decomposição microbiana de resíduos orgânicos, e tem recebido atenção especial como um dos fatores limitantes na criação intensiva dos peixes (Tomasso, 1994). Silva e Fukimoto (2015) em um experimento com recria de tambaquis em tanques rede, em um lago de 0,55 ha, abastecido com água de chuva em profundidade máxima de $2,5 \mathrm{~m}$, também verificou que não houve alteração nos parâmetros de nitrogênio e fósforo total, que pudessem causar eventos de eutrofização naquele ambiente.

Os resultados revelados no presente estudo, demostram que a área onde foram implantados os tanques redes $(0,25$ ha de lâmina d'água), não apresentou alterações significativas dos parâmetros indicadores de eutrofização, como o nitrogênio e fósforo total, e em nenhum outro parâmetro físico-químico analisado. Nesse sentido, se for projetado o cultivo de tambaquis em tanques rede para 1,0 ha de lâmina d'água, pode-se quadruplicar o número de tanques rede, sem que haja o desequilíbrio nas variáveis indicadoras de eutrofização, devido principalmente, a uma maior capacidade de suporte do ambiente em diluir tais nutrientes. Segundo Feiden et al. (2015), os danos causados pela criação de peixes dependem de vários fatores, em especial a capacidade do ambiente em diluir e reciclar os dejetos e restos de ração oriundos dos cultivos, e da capacidade de suporte do ambiente, fatores que também foram corroborados no presente estudo.

\section{ASSEMBLEIAS DE PEIXES}

Alterações nos índices descritores de comunidades muitas vezes podem estar associadas as modificações provocadas no ambiente (Magurran, 1988). Como por exemplo, o aumento dos níveis de estresse ambiental, geralmente tem sido relacionado ao decréscimo da diversidade, da riqueza específica e da equitabilidade, com o consequente incremento da dominância de espécies. Outros estudos também demostram que espécies de peixes em ambientes naturais, muitas vezes podem se beneficiar da criação de peixes em tanques redes, tanto em ambiente marinho (Dempster et al., 2003; Boyra et al., 2004), como de água doce (Carvalho, 2006; Ramos et al., 2008a; Ramos, 2009).

No presente estudo, uma maior riqueza (14 spp.), diversidade $\left(H^{\prime}=2,05\right)$ e abundância (262 indivíduos) da ictiofauna foi encontrada próximo aos tanques rede, indicando uma provável mudança na dinâmica ecológica local em vista da presença dos tanques rede. Esse padrão ecológico das espécies de peixes pode estar relacionados a vários fatores: I) As espécies de peixes estão se beneficiando das sobras de alimentos (ração) colocados para os peixes cultivados (ex.: Bryconops alburnoides, Colossoma macropomum, Metynnis argenteus, Metynnis hypsauchen e Geophagus altifrons), essas espécies possuem hábito alimentar onívoro (Soares et al., 2007; Zuanon et al., 2015) o que reforça o argumento de que elas podem estar frequentando a área próximas aos tanques rede, a procura destes resíduos alimentares; II) as espécies de pequeno porte (comprimento padrão $<10 \mathrm{~cm}$ ) podem estar utilizando os tanques rede, como local de abrigo contra predadores, como por exemplo, Bryconops alburnoides, Bryconops giacopinii, Moenkhausia lepidura e Brycon pesu (observação pessoal), uma vez que esses pequenos indivíduos passam facilmente entre as malhas dos tanques rede; III) algumas espécies estão se alimentando das fezes dos peixes cultivados, como exemplo, Hemiodus argenteus, peixe com hábito alimentar detritivívoro (Soares et al., 2007) e, IV) Peixes predadores (ex.: Acestrorhynchus microlepis, Serrasalmus rhombeus, Agoniates halecinus, Cichla monoculus e Plagioscion squamosissimus) com hábito alimentar piscívoro (Soares et al., 2007) foram abundantes no sitio onde estavam instalados os tanques rede, os quais, certamente podem ser atraídos pela grande abundância de espécies de pequeno porte, disponíveis naquele local. 
Estudos realizados por Ramos et al. (2008a) e Ramos (2009) em represas do rio Tietê (Sudeste do Brasil) também observaram espécies de peixes agregando-se aos sistemas de piscicultura em tanques rede, criando novas teias alimentares, sustentadas pelo aporte de efluentes e sobras de rações. Carvalho (2006) e Carvalho et al. (2008), demostraram que a piscicultura em tanques rede pode afetar de diferentes formas a ictiofauna residente, tanto na riqueza de espécies quanto na diversidade.

Outros estudos realizados por Paes (2006), Zanata (2007) e Ramos et al. (2008a), sobre os impactos dos tanques rede na ictiofauna em represas do Sudeste do Brasil, também revelam uma maior diversidade de espécies em torno dos tanques rede, com a dominância de poucas espécies. Estes fatos de atratividade dos tanques rede são bastante semelhantes aos estudos em ecossistemas marinhos costeiros, como indicados em estudos por Dempster et al. (2003) e Boyra et al. (2004). Interferência induzida por esse tipo de atividade podem levar a mudanças nos hábitos alimentares dos peixes nativos (Ramos et al., 2008b) e isso pode levar a longo prazo a interferir na dinâmica ecológica local, visto que, pode haver alteração da dieta de muitas espécies de peixes, o que pode estabelecer novas relações ecológicas na biota (Echer, 2008).

\section{Conclusões}

Os resultados demostram que, houve uma atratividade das espécies de peixes as áreas próximas aos tanques rede, ocorrentes durante o período de estudo. Essa característica verificada em um lago de barragem na Amazônia é semelhante o que ocorreu em outros ambientes de água doce e marinha do Brasil. Assim, a piscicultura em tanques redes em ambientes lênticos abertos, como o reservatório da hidrelétrica de Balbina, certamente pode a longo período de tempo interferir na dinâmica das assembleias de peixes residentes. Contudo, para essa afirmativa, ressalta-se que estudos com um período de monitoramento maior durante o cultivo dos peixes em taques redes devem ser realizados em ambientes lênticos na Amazônia.

\section{Referências}

Agostinho, A. A., Gornes, L. C. \& Peicice, E. F. M. (2007). Ecologia e manejo de recursos pesqueiros em reservatórios do Brasil. Maringá-PR: Editora EDUEM.

Boyra, A., Sanchez-Jerez, P., Tuya, F.; Espino, F. \& Haroun, R. (2004). Attraction of wild costal fishes to na Atlantic subtropical cage fish farms, Gran Canária, Canary Islands. Enviromental Biology of Fishes, 70(2): 393-401.

Brabo, M. F.; Vera, G. C.; Paiva, R. S.; Fujimoto, R. Y. (2014). Aproveitamento aquícola dos grandes reservatórios brasileiros. Boletim Instituto de Pesca, 40(1): 121-134.

Bueno, G. W., Matos, F. T., Canzi, C., Ostrensky, A., Sampaio, M. B., Barone, R. S. C. \& Roubach, R. (2011). Produção de peixes cultivados em reservatórios: a capacidade de suporte. Revista Panorama da Aquicultura, 21(126): 48-63.

Carvalho, E. D. (2006). Avaliação dos impactos da piscicultura em tanques rede nas represas dos grandes tributários do Alto Paraná (Tietê e Paranapanema): o pescado, a ictiofauna agregada e as condições limnológicas. Botucatu, SP: Instituto de Biociência - Universidade Estadual Paulista.

Carvalho, E. D., Silva, I. P., Ramos, D. M. M. \& Rezende-Ayroza, L. M. (2008). Caracterização das condições limnológicas juntos aos sistemas de tilapicultura em tanques rede no reservatório da U.H.E. de Chavantes, médio rio Paranapanema. Botucatu, SP: Instituto de Biociência- Universidade do Estadual Paulista.

Carvalho, E. D. \& Ramos, I. P. A. (2010). Aquicultura em grandes represas brasileiras: interfaces ambientais, socioeconômicas e sustentabilidade. Boletim da Sociedade Brasileira de Limnologia, 38(1): 49-57.

Conama. Conselho Nacional do Meio Ambiente. Resolução no 357, de 17 de março de 2005. Dispõe sobre a classificação dos corpos de água e diretrizes ambientais para o seu enquadramento, bem como estabelece as condições e padrões de lançamento de efluentes, e dá outras providências. Brasília: Diário Oficial da União, 2005 .

Dempster, T., Sachez-Jerez, P., Bayle-Sempere, J. T., Giménez-Casalduero, F. \& Valle, C. (2003). Atractiono of wild fish to sea-cage fish farms in the South-Westem Mediterrnean Sea: spatial and short-term temporal variability. Marine Ecology Progress Series, 242(1): 237-252. 
Eche, L. M. F. (2008). Cultivo de peixes em tanques rede: efeito sobre a energia e a estrutura trófica em ambientes aquáticos. [Dissertação de Mestrado]. Maringa (PR): Universidade Estadual de Maringá.

FAO. Perspectivas de la agricultura y del desarrollo rural en las Américas: una mirada hacia América Latina y el Caribe 2017-2018 /CEPAL, FAO, IICA, 2017.

Fearnside, P. M. (2012). Hidrelétricas amazônicas e a política energética - Parte 1. Acessado em: http://colunas.globoamazonia.com/philipfearnside/

Fearnside, P. M. (2015). A Hidrelétrica de Balbina: O faraonismo irreversível versus o meio ambiente na Amazônia. In: Fearnside, P. M. Hidrelétricas na Amazônia: Impactos Ambientais e Sociais na Tomada de Decisões sobre Grandes Obras. Manaus-AM: Editora INPA.

Feiden, I. F.,Oliveira, J. D. S., Diemer, O. \& Feiden, A. (2015). Qualidade da água, capacidade de suporte e melhor período para criação de peixes em tanques-rede no reservatório de Salto Caxias. Engenharia Sanitária e Ambiente, 20(4): 589-594.

Guarino, A. W. S., Branco, C. W. C., Diniz, G. P. \& Rocha, R. (2005). Limnological characteristics of an old tropical reservoir (Ribeirão da Lages Reservoir, RJ, Brazil). Acta Limnologica Brasiliensia, Botucatu, 17(2): 129-141.

Guo, L., Li, Z., Xie, P. \& Ni, L. (2009). Assessment effects of cage culture on nitrogen and phosphorus dynamics in relation to fallowing in a shallow lake in China. Aquaculture International, 17(1): 229-241.

Krebs, C. J. (1989). Ecological Methodology. Benjamin/Cummings.

Lees, A. C., Peres, C. A., Fearnside, F. M., Schneider, M. \& Zuanon, J. A. S. (2016). Hydropower and the future of Amazonian biodiversity. Biodiversity and Conservation, 25(1): 451-466. Doi 10.1007/s10531-016$1072-3$

Magurran, A. E. (1988). Ecological Diversity and Its Measurement. New York: Chapman and Hall.

Mallasen, M., Carmo, C. F., Tucci, A., Barros, H. P., Rojas, N. E. T., Fonseca, F. S. \& Yamashita, E. Y. (2012). Qualidade da Água em Sistema de Piscicultura em Tanques rede no Reservatório de Ilha Solteira, SP. Boletim Instituto de Pesca, 38(1): 15-30.

Ono, E. A. (2005). Cultivar peixes na Amazônia: Possibilidade ou Utopia? Panorama da aquiicultura, 15(1): 41-48.

Ono, E. A. \& Kubitza, F. (2003). Cultivo de peixes em tanque-rede. Jundiaí-SP: Editora Degaspari.

Paes, J. V. K. (2006). A ictiofauna associada e as condições limnológicas numa área de inflência da criação de tilápia em tanques rede no reservatório de Nova Avanhandava. [Dissertação de Mestrado]. Botucatu (SP): Universidade Estadual Paulista.

Ramos, I. P. (2009). Aspectos da biologia populacional de Pimelodus maculatus (Teleostei: Siluriformes), sob influência de sistemas de piscicultura em tanques rede. [Dissertação de Mestrado]. Botucatu (SP):Universidade Estadual Paulista.

Ramos, I. P., Zanata, A. S., Zica, E. O. P., Silva, R. J. \& Carvalho, E. D. (2008). Impactos ambientais de piscicultura em tanques rede sobre águas continentais brasileiras: revisão e opinião. In: Cyrino, J. E. P., Furuyaw, M., Ribeiro, R. P. \& Scorvo-Filho, J. D. (Eds.). Tópicos especiais em biologia aquática e aquicultura III. Jaboticabal: Editora Sociedade Brasileira de Aquicultura e Biologia Aquática. (a)

Ramos, I. P., Vidotto-Magnoni, A. P. P. \& Carvalho, E. D. (2008). Influence of cage fish farming on the diet of dominant fish species of a Brazilian reservoir (Tietê River, High Paraná River basin). Acta limnologica Brasiliesia, 20(3): 245-252. (b)

Silva, C. A. \& Fujimoto, R. Y. (2015). Crescimento de tambaqui em resposta a densidade de estocagem em tanques rede. Acta Amazonica, 45(3): 323-332.

Soares, M. G. M., Costa, E. L., Siqueira-Souza, F. K., Anjos, H. D. B., Yamamoto, K. C. \& Freitas, C. E. C. (2007). Peixes de lagos do médio Rio Solimões. Manaus: Editora EDUA. 
Tomasso, J. R. (1994). Toxicity of nitrogenous wastes to aquaculture animals. Reviews in Fisheries Science, 2(1): 291-314.

Tundisi, J. G. \& Matsumura-Tundisi, T. (2003). Integration of research and management in optimizing multiple uses of reservoirs: the experience of South America and Brazilian cases studies. Hydrobiologia, 500(3): 231-242.

Winemiller, K. O., Mcintyre, P. B., Castello, L., Fluet-Chouinard, E., Giarrizzo, T., Nam, S., Baird, I. G., Darwall, W., Lujan, N. K., Harrison, I. \& Stiassny, M. L. (2016). Balancing hydropower and biodiversity in the Amazon, Congo, and Mekong. Science, 351: 128-129.

Zanata, A. S. (2007). Tilapicultura em ecossistemas aquáticos: Desenviolvimento sustentável ou degradação ambiental? Estuds de caso em represa oligotrófica. [Dissertação de Mestrado]. Botucatu (SP): Universidade Estadual Paulista.

Zar, J. H. (2009). Biostatistical analysis. Prentice-Hall: Editora Englewood cliffs.

Zuanon, J., Mendonça, F. P., Espirito-Santo, H. M., Dias, M. S., Galuch, A. V. \& Akama, A. (2015). Guia de Peixes da Reserva Ducke, Amazônia Central. Manaus: Editora INPA. 